\title{
ON THE HOPF-TODA INVARIANT
}

\author{
BY
}

K. A. HARDIE

0 . Introduction. One line of attack on the problem of computing the unstable homotopy groups of spheres is to attempt to construct the elements whose reduced product filtration exceeds 1 . The classical Hopf construction associates with a map $S^{m} \times S^{t-1} \rightarrow S^{n}$ of type $(\alpha, \beta)$ an element of $\pi_{m+t}\left(S^{n+1}\right)$. The indeterminacy of the construction is the suspension subgroup in the sense that the elements associated with any two maps of the same type differ by a suspension. However, it was proved by I. M. James [8] that the filtration of the element obtained does not exceed 2. In [3] I defined a generalization of the Hopf construction which can yield elements of arbitrarily large filtration. Thus, for example, let $\partial$ denote the homotopy boundary homomorphism and let $\alpha \in \pi_{m}\left(S_{r-2}^{n}\right)$, $\beta \in \pi_{t}\left(S_{r-1}^{n}, S_{r-2}^{n}\right)$ be elements $\left({ }^{1}\right)$ such that the Whitehead product $[\alpha, \partial \beta]$ is trivial. Then there exists a map $F: S^{m} \times S^{t-1} \rightarrow S_{r-2}$ of type $(\alpha, \partial \beta)$. Applying the construction we obtain an element $c(F) \in \pi_{m+t}\left(S^{n+1}\right)$ with indeterminacy equal to the subgroup of filtration $r-2$. As always the problem is to prove nontriviality. To this end we study a homomorphism

$$
H: \pi_{i}\left(S_{r-1}^{n}, S_{r-2}^{n}\right) \rightarrow \pi_{i+1}\left(S^{r n}\right)
$$

equivalent to a special case of the "relative Hopf homomorphism" of H. Toda [14]. If $r=2$ then $H$ reduces to the generalized Hopf invariant $H^{*}$ of P. J. Hilton [5]. Our main theorem may be stated as follows.

Let $n$ be even and let $r=p^{s}$ be a power of a prime $p \geqq 2$. Let $h_{r-1}: S_{r-1}^{n}, S_{r-2}^{n} \rightarrow S^{(r-1) n}, *$ be a map of degree 1 , let $E$ denote suspension and suppose that $\left(h_{r-1}\right)_{*} \beta=E \beta^{\prime \prime}$. Let

$$
\tau=E^{(r-1) n-1}(\phi i x) \circ E^{m+1} \beta^{\prime \prime} \in \pi_{m+t}\left(S^{r n}\right),
$$

where $\phi: \pi_{m}\left(S_{\infty}^{n}\right) \rightarrow \pi_{m+1}\left(S^{n+1}\right)$ denotes the James canonical isomorphism [6], and $i$ is induced by the injection $S_{r-2}^{n} \rightarrow S_{\infty}^{n}$. Let $\{c(F)\}$ denote the coset of $c(F)$ in $\pi_{m+t}\left(S^{n+1}\right) / \phi i \pi_{m+t-1}\left(S_{r-2}^{n}\right)$. Then we have:

THEOREM 0.2. (a) If (i) $\tau$ is of order $p^{w}$, (ii) $\tau=p^{k} E^{2} \theta$, where $\theta \in \pi_{m+t-2}\left(S^{r n-2}\right)$ and $E^{3} \theta$ generates $\pi_{m+t+2}\left(S^{r n+1} ; p\right)$, then $\{c(F)\}$ is of order $p^{v}$, where $v \geqq \min (s-k, w)$.

Received by the editors October 22, 1962.

(1) $S_{r}^{n}$ denotes the $r n$-skeleton of the reduced product complex $S_{\infty}^{n}$ of the $n$-sphere. 
(b) If $\tau=p^{k} \iota_{r n}$ then $\{c(F)\}$ is of order $p^{v}$, where $v \geqq s-k$.

For example, using the formula 4.4 due to Toda it is easy to prove that $\left[\iota_{2},\left[\iota_{2}\right]^{p-1}\right]=0 \in \pi_{2 p-2}\left(S_{p-2}^{2}\right)$. Applying $0.2(\mathrm{~b})$ we recover the nonzero elements of $\pi_{2 p}\left(S^{3}\right)$. Our main application of 0.2 is however as follows. Let $\alpha_{t} \in \pi_{2 t(p-1)+2}\left(S^{3}\right)(t \geqq 1)$ be the elements of order $p$ described by Toda in [16]. Then we prove for all $m+t \leqq p$ the existence of maps of type

$$
\left(\iota_{2 m},\left[\iota_{2 m}\right]^{p-1} \circ E^{2 m(p-1)-4} \alpha_{t}\right)
$$

whose Hopf construction elements are nontrivial. Using the computations due to Toda of the stable groups we are able to compute the orders of the $p$-components of the unstable groups of the $q$-stem for $q<2 p(p-1)$. Let $p$ be an odd prime and let $[r, n]$ denote the $p$-primary component of $\pi_{r}\left(S^{n}\right)$. We obtain $\left({ }^{2}\right)$

THEOREM 0.3 .

(a) $[2 t(p-1)+2 m-s, 2 m+1]=0 \quad(m \geqq 1,1 \leqq t \leqq p, 2 \leqq s \leqq 2 p-3)$;

(b) (i) $[2 t(p-1)+2 m-1,2 m+1]=Z_{p} \quad(1 \leqq t \leqq p-1,1 \leqq m \leqq t-1)$;

(ii) $[2 t(p-1)+2 m-1,2 m+1]=0(1 \leqq t \leqq p-1, m \geqq t)$;

(iii) $[2 p(p-1)+1,3]=Z_{p}$;

(iv) $\left.\begin{array}{rl}{[2 p(p-1)+2 m-1,2 m+1]} & =Z_{p^{2}} \text { or } Z_{p}+Z_{p} \quad(2 \leqq m \leqq p-1) \\ & =Z_{p} \quad(m \geqq p)\end{array}\right\}$;

(c) (i) $[2 t(p-1)+2 m, 2 m+1]=Z_{p} \quad(1 \leqq t \leqq p-1, m \geqq 1)$;

(ii) $[2 p(p-1)+2 m, 2 m+1]=Z_{p^{2}} \quad(m \geqq 2)$;

(iii) $[2 p(p-1)+2,3]=Z_{p}$.

1. The relative Hopf invariant. Let $K=K_{0} \cup e^{r}$ be a finite cell complex which is the result of attaching an $r$-cell $e^{r}$ to the subcomplex $K_{0}$. Let $\theta: K \rightarrow K \vee S^{r}$ be a map which shrinks an $(r-1)$-sphere contained in $e^{r}$, is such that the composition of $\theta$ with the projection on to $S^{r}$ is a map of degree one and such that the composition of $\theta$ with the projection on to $K$ is homotopic to the identity map. Then we recall that the relative Hopf invariant due to Toda [14] is a homomorphism $H^{\prime}$ such that the diagram

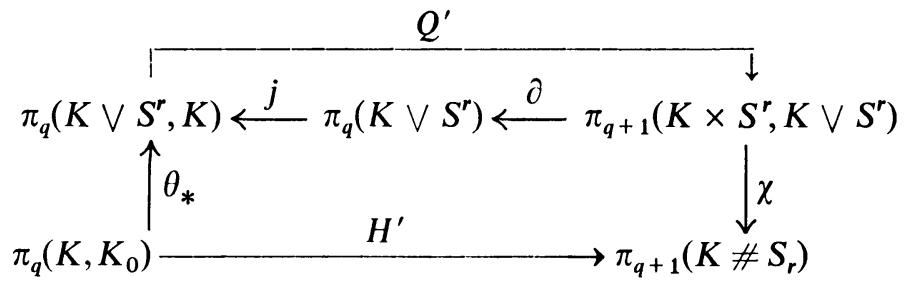

is commutative where $j$ is an injection, $\partial$ is the homotopy boundary, $\chi$ is induced by the collapsed product identification and $Q^{\prime}$ is a left inverse of $j \partial$.

(2) 0.3 agrees with and slightly extends the recent computations of Toda in Composition methods, Annals of Mathematics Studies No. 49, Princeton Univ. Press, Princeton, N. J.; H. H. Gershenson has proved that the possibility $Z_{p}+Z_{p}$ in (b) (iv) can be deleted. 
Let $V^{n}$ and $S^{n}$ denote the $n$-element and $n$-sphere as represented in [7]. We shall assume that orientations for $V^{n}, S^{n}$ and products have been chosen as in [7]. Then the homotopy boundary homomorphism $\partial: \pi_{n}(X, A) \rightarrow \pi_{n-1}(A)$ is defined accordingly. Let $S$ denote the reduced suspension function in the category of based spaces and based maps. Let $\iota_{1}$ be the preferred generator of $\pi_{1}\left(S^{1}\right)$ so that if $\gamma \in \pi_{n}(X)$ then

$$
E \gamma=\gamma \# \iota_{1} \in \pi_{n+1}\left(X \# S^{1}\right)=\pi_{n+1}(S X) .
$$

If $\alpha \in \pi_{t-1}\left(S^{q-1}\right)$ and $\alpha^{\prime} \in \pi_{t}\left(V^{q}, S^{q-1}\right)$ are elements such that $\alpha=\partial \alpha^{\prime}$ then, again following James, we shall denote by $\alpha^{*}: \pi_{q-1}(X) \rightarrow \pi_{t-1}(X)$ and $\alpha^{* *}: \pi_{q}(X, A) \rightarrow \pi_{t}(X, A)$ the operators determined by composition with $\alpha$ and $\alpha^{\prime}$. We recall that

$$
\alpha^{* *}=(-1)^{q+t}(E \alpha)^{*}: \pi_{q}(X, *) \rightarrow \pi_{t}(X, *)
$$

It is well known [17] that $\alpha^{*}$ and $\alpha^{* *}$ are homomorphisms if $\alpha$ is a suspension element. The following lemma is easily proved using 1.1 and the definition of $H^{\prime}$.

LemMA 1.2. $H^{\prime} \alpha^{* *}=\left(E^{2} \alpha\right)^{*} H^{\prime}$.

Let $h: K, K_{0} \rightarrow S^{r}, *$ be a map of degree one on $e^{r}$. Applying an argument essentially due to Toda we obtain the following theorem in which the square brackets refer to the Blakers-Massey generalized Whitehead product defined in [1]. Let $i: \pi_{n}\left(K_{0}\right) \rightarrow \pi_{n}(K)$ denote the injection.

THEOREM 1.3. If $\alpha \in \pi_{m}\left(K_{0}\right)$ and $\beta \in \pi_{t}\left(K, K_{0}\right)$ then

$$
H^{\prime}[\alpha, \beta]=(i \alpha) \# h_{*} \beta \in \pi_{m+t}\left(K \# S^{r}\right) .
$$

Proof. Let $*$ denote the product defined in $[1 ; 5]$. Then we have $\chi\left(i \alpha * h_{*} \beta\right)$ $=i \alpha \# h_{*} \beta$. In view of the commutativity of the diagram it is thus sufficient to prove that $j \partial\left(i \alpha * h_{*} \beta\right)=\theta_{*}[\alpha, \beta]$. This however follows from $[1,5.7,3.10]$ and the definition of $\theta$.

Now suppose that $\alpha$ and $\beta$ are such that $[\alpha, \partial \beta]=0 \in \pi_{m+t-2}\left(K_{0}\right)$. Then there is a map $F: S^{m} \times S^{t-1} \rightarrow K_{0}$ of type $(\alpha, \partial \beta)$. Let

$$
F^{\prime}:\left(S^{m} \times S^{t-1}\right) \cup\left(* \times V^{t}\right) \rightarrow K
$$

be an extension of $F$ such that $F^{\prime}(*, x)=g(x)\left(x \in V^{t}\right)$ where $g: V^{t}, S^{t-1} \rightarrow K, K_{0}$ represents $\beta$. Let $\psi$ be a characteristic map for the $(m+t)$-cell of $S^{m} \times V^{t}$ and let

$$
\gamma=\left\{F^{\prime} \psi \mid S^{m+t-1}\right\} \in \pi_{m+t-1}(K) .
$$

Then we have the theorem:

THEOREM 1.6. $j \gamma=[\alpha, \beta] \in \pi_{m+t-1}\left(K, K_{0}\right)$.

Proof. Let $E_{+}^{t-1}$ and $E_{-}^{t-1}$ be the 'Northern' and 'Southern' hemispheres of $S^{t-1}$. We can certainly replace $F$ by a homotopic map with the property that 


$$
F(x, y)=F(x, *) \text { if }(x, y) \in S^{m} \times E_{+}^{t-1} \text {. }
$$

Then the injection $V^{t} \rightarrow\left(S^{m} \times S^{t-1}\right) \cup\left(* \times V^{t}\right)$ followed by $F^{\prime}$ is a map $\left(V^{t}, S^{t-1}, E_{+}^{t-1}\right) \rightarrow\left(K, K_{0}, *\right)$. Let $\psi_{m}:\left(V^{m}, S^{m-1}\right) \rightarrow\left(S^{m}, *\right)$ be a map of degree one and let $\left(V^{m} \times V^{t}\right)^{\cdot}=\left(S^{m-1} \times V^{t}\right) \cup\left(V^{m} \times S^{t-1}\right)$. Then $\phi:\left(V^{m} \times V^{t}\right)^{\cdot} \rightarrow K$ represents $\gamma$ where

$$
\phi=F^{\prime}\left(\left(\psi_{m} \times 1\right) \mid\left(V^{m} \times V^{t}\right)^{\cdot}\right) .
$$

However, if $A=\left(S^{m-1} \times V^{t}\right) \cup\left(V^{m} \times E_{+}^{t-1}\right)$ then it follows from the definition $[1,3.1]$ that $\phi \mid A$ represents $[\alpha, \beta] . V^{m} \times E_{-}^{t-1}$ is the face of $V^{m} \times V^{t}$ complementary to $A$ and $\phi\left(V^{m} \times E_{-}^{t-1}\right) \subseteq K_{0}$. Since $A$ and $\left(V^{m} \times V^{t}\right)^{\cdot}$ are required to be oriented coherently, 1.6 follows from $[10,22.1]$.

2. Reduced products. Let $A$ be a countable $\mathrm{CW}$-complex with but one 0 -cell at the base-point $*$. The reduced product complex $A_{\infty}[6]$ is a space whose points are equivalence classes of finite sequences of points of $A$. Let $A_{n}(n \geqq 0)$ denote the subcomplex determined by the sequences with $n$ terms or fewer, $A_{0}$ being identified with * and $A_{1}$ with $A$. Let $\Omega$ be the space of based loops on $S A$, let $\sigma: S \Omega \rightarrow S A$ be the map such that $\sigma(x, t)=x(t)$ and let $\phi_{1}: A_{\infty} \rightarrow \Omega$ be a canonical map in the sense of [6]. Then if $\psi=\sigma S\left(\phi_{1}\right)$ we recall that

$$
\phi=\psi_{*} E: \pi_{q}\left(A_{\infty}\right) \rightarrow \pi_{q+1}(S A)
$$

is the canonical isomorphism of James. We shall also denote by $\psi$ the maps from $S\left(A_{n}\right)$ to $S(A)$ which agree with $\psi$.

Let $\alpha \in \pi_{m}\left(A_{n}\right), \quad \beta^{\prime} \in \pi_{t-1}\left(A_{n}\right)$ be elements whose. Whitehead product $\left[\alpha, \beta^{\prime}\right] \in \pi_{m+t-2}\left(A_{n}\right)$ vanishes. Then there is a map $F: S^{m} \times S^{t-1} \rightarrow A_{n}$ of type $\left(\alpha, \beta^{\prime}\right)$. The generalization of the Hopf construction described in [3] associates with $F$ the element

$$
c(F)=(\psi S(F) \eta)_{*} \iota \in \pi_{m+t}(S A),
$$

where $\iota$ denotes the orientation class of $S\left(S^{m} \# S^{t-1}\right)$ and $\eta$ is a homotopy right inverse of the suspension of the collapsed product identification $\chi: S^{m} \times S^{t-1} \rightarrow S^{m} \# S^{t-1}$.

$\pi_{q}\left(A_{\infty}\right)$ is filtered by the subgroups $i \pi_{q}\left(A_{n}\right)$ and the reduced product filtration in $\pi_{q+1}(S A)$ is that induced by $\phi$. The following lemma (on which we shall not rely) is a consequence of the definition 2.2 .

LEMMA 2.3. If $\sigma \in \pi_{m+t}(S A)$ then there exists a map $G: S^{m} \times S^{t-1} \rightarrow A_{n}$ of type $\left(\alpha, \beta^{\prime}\right)$ such that $c(G)=\sigma$ if and only if the filtration of $\sigma-c(F)$ does not exceed $n$.

Now let $A=S^{n}, K=S_{r-1}^{n}, K_{0}=S_{r-2}^{n}$. Then $H^{\prime}$ as defined in 1 is a homomorphism 


$$
H^{\prime}: \pi_{q}\left(S_{r-1}^{n}, S_{r-2}^{n}\right) \rightarrow \pi_{q+1}\left(S_{r-1}^{n} \not \# S^{(r-1) n}\right) .
$$

Let

$$
H=\lambda_{*} H^{\prime}: \pi_{q}\left(S_{r-1}^{n}, S_{r-2}^{n}\right) \rightarrow \pi_{q+1}\left(S^{r n}\right),
$$

where $\lambda$ is the map defined by the diagram

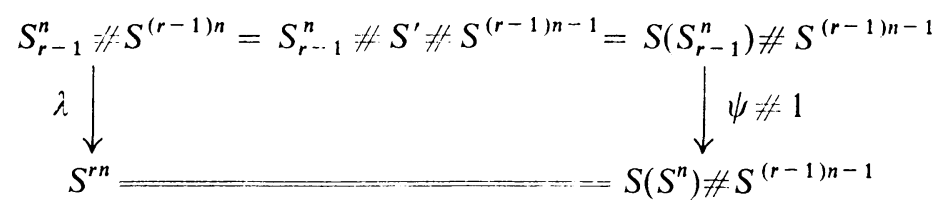

in which the equalities denote orientation preserving homeomorphisms and $\psi \# 1$ the collapsed product map. Let $\alpha \in \pi_{m}\left(S_{r-2}^{n}\right), \beta \in \pi_{t}\left(S_{r-1}^{n}, S_{r-2}^{n}\right)$ then we have:

THEOREM 2.5. If $h_{*} \beta=E \beta^{\prime \prime}$, where $\beta^{\prime \prime} \in \pi_{t-1}\left(S^{(r-1) n-1}\right)$ then

$$
H[\alpha, \beta]=(-1)^{k} E^{(r-1) n-1}(\phi i \alpha) \circ E^{m+1} \beta^{\prime \prime},
$$

where $k=n(m+1)(r-1)+m t-1$.

Proof. Applying 1.3, 2.1 and finally $[7,3.5]$ we obtain

$$
\begin{aligned}
H[\alpha, \beta] & =\lambda_{*}\left(i \alpha \not-\beta^{\prime \prime} \not \# \iota_{1}\right) \\
& =(-1)^{t-1} \lambda_{*}\left(i \alpha \neq \iota_{1} \not \# \beta^{\prime \prime}\right) \\
& =(-1)^{t-1}(\phi i \alpha) \not \# \beta^{\prime \prime} \\
& =(-1)^{k} E^{(r-1) n-1}(\phi i \alpha) \circ E^{m+1} \beta^{\prime \prime} .
\end{aligned}
$$

Now suppose that $F: S^{m} \times S^{t-1} \rightarrow S_{r-2}^{n}$ is a map of type $(\alpha, \partial \beta)$ and let $\gamma \in \pi_{m+1-1}\left(S_{r-1}^{n}\right)$ be defined by 1.5 . Then we assert that

$$
\phi i \gamma= \pm c(F) \text {. }
$$

A proof of a special case can be found in $\left[4\right.$, p. 248-9] $\left({ }^{3}\right)$. The argument is quite general, however, and in the present situation yields 2.6.

Let $h_{r}: S_{r}^{n}, S_{r-1}^{n} \rightarrow S^{r n}$, * be a map of degree 1 . We shall also denote by $h_{r}$ any map and any homomorphism induced by any map which agrees with a cellular approximation of the combinatorial extension $h_{r}: S_{\infty}^{n} \rightarrow S_{\infty}^{r n}$ of $h_{r}$. (See $\left[6\right.$, p. 173].) Let $p$ be a prime and let $\mathscr{C}_{p}$ denote the class of all finite Abelian groups of order prime to $p$. We shall require the following result of [2].

LEMma 2.7. If $n$ is even and $r=p^{q}$ then there are $\mathscr{C}_{p}$-isomorphisms

$$
h_{r}: \pi_{i}\left(S_{\infty}^{n}, S_{r-1}^{n}\right) \rightarrow \pi_{i}\left(S_{\infty}^{r n}\right) \quad(i>0) .
$$

(3) There is a misprint in [4, p. 249, line 5]: $\left(k^{\prime \prime}\right)_{* l}$ generates the summand $\pi_{2 p}\left(S^{2 p}\right)$. 
3. The $E-H-Q$ sequence. Let $n$ be even, let $r$ be a power of a prime $p \geqq 2$. Then, in view of $2.7, \phi h_{r}: \pi_{q}\left(S_{\infty}^{n}, S_{r-1}^{n}\right) \rightarrow \pi_{q+1}\left(S^{r n+1}\right)$ certainly defines an isomorphism of the $p$-components. Let $\mu_{t}$ denote the characteristic class of the $t n$-cell of $S_{t}^{n}$ and also its image under injection in $\pi_{t n-1}\left(S_{\infty}^{n}, S_{t-1}^{n}\right)$. Let $k: \pi_{q+1}\left(S^{r n+1}\right) \rightarrow \pi_{q}\left(S_{\infty}^{n}, S_{r-1}^{n}\right)$ be the homomorphism such that

$$
\begin{aligned}
k \alpha & =\left(\phi h_{r}\right)^{-1} \alpha & & \text { if the order of } \alpha \text { is a power of } p, \\
& =0 & & \text { if } \alpha \text { is of finite order prime to } p, \\
& =\mu_{r} & & \text { if } \alpha=\iota_{r n+1} .
\end{aligned}
$$

Then since $\pi_{q+1}\left(S^{r+1}\right)$ is finite unless $q=r n, k$ is a $\mathscr{C}_{p}$-isomorphism. Let $\mathscr{C}$ be a class of Abelian groups. We recall that a sequence $G_{1} \stackrel{\phi_{2}}{\rightarrow} G_{2} \stackrel{\phi_{2}}{\rightarrow} G_{3}$ is $\mathscr{C}$-exact at $G_{2}$ in the sense of Spanier [12] if $\phi_{2} \phi_{1}=0$ and if the homology group at $G_{2}$ is in $\mathscr{C}$. Let $H_{r}=\phi h_{r} j \phi^{-1}, Q_{r}=\partial k$ and let $E_{r}=\phi i$. It follows easily that the sequence

$$
\rightarrow \pi_{q}\left(S_{r-1}^{n}\right) \stackrel{E_{r}}{\longrightarrow} \pi_{q+1}\left(S^{n+1}\right) \stackrel{H_{r}}{\longrightarrow} \pi_{q+1}\left(S^{r+1}\right) \stackrel{Q_{r}}{\longrightarrow} \pi_{q-1}\left(S_{r-1}^{n}\right) \rightarrow
$$

is $\mathscr{C}_{p}$-exact.

Let $\alpha \in \pi_{q-1}\left(S^{r n-1}\right)$ be of infinite order or else of order a power of $p$. We require the lemma:

LEMMA 3.3. $Q_{r} E^{2} \alpha=(-1)^{q}\left[\iota_{n}\right]^{r} \circ \alpha$.

Proof. In the following diagram, $i^{\prime}$ is an injection, $\theta$ a map of degree 1 and $P=\left(\left[\iota_{n}\right]^{r}\right)_{*}$.

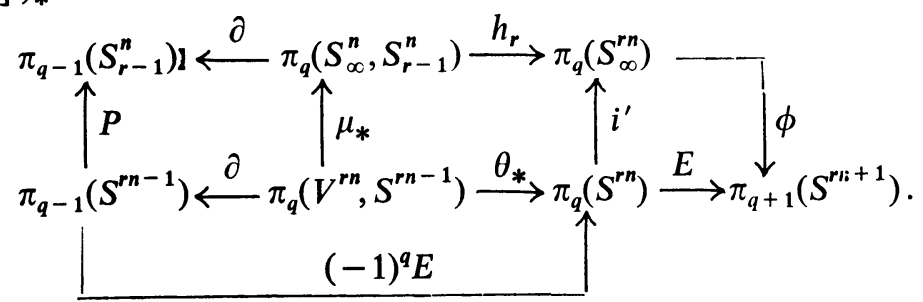

The lower rectangle is commutative, for let $\beta^{\prime} \in \pi_{q}\left(V^{r n}, S^{r n-1}\right)$ and let $\beta=\partial \beta^{\prime}$. Then $\theta_{*} \beta^{\prime}=\beta^{* *} \iota_{r n}=(-1)^{q}(E \beta)^{*} \iota_{r n}=(-1)^{q} E \beta$. The other portions of the diagram are also commutative and the lemma follows from the definition of $Q_{r}$.

Let $\Delta$ be defined by the commutative diagram

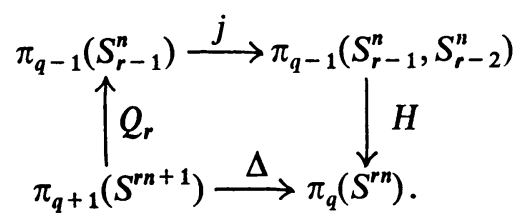

By $\left[14,5.9^{\prime \prime}\right]$ we have $j\left[\iota_{n}\right]^{r}=r\left[\iota_{n}, \mu_{r-1}\right]$ and hence applying $3.3,1.2$ and 2.5 we obtain: 
LEMMA 3.5. If $\gamma \in \pi_{q-2}\left(S^{r n-2}\right)$ is of infinite order or else of order a power of $p$ then $\Delta E^{3} \gamma=-r E^{2} \gamma$.

Proof of Theorem 0.2. Let $i^{\prime}$ denote the injection $\pi_{m+t-1}\left(S_{r-2}^{n}\right) \rightarrow \pi_{m+t-1}\left(S_{r-1}^{n}\right)$. Then by the exactness of the homotopy sequence of the pair $\left(S_{r-1}^{n}, S_{r-2}^{n}\right)$ we have

$$
\text { kernel } j=i^{\prime} \pi_{m+t-1}\left(S_{r-2}^{n}\right) \text {. }
$$

Moreover, by the $\mathscr{C}_{p}$-exactness of the $E-H-Q$ sequence we have $p^{v} \gamma \in$ kernel $E_{r}$ if and only if $p^{v} \gamma \in Q_{r} \pi_{m+t+1}\left(S^{r n+1}\right)$. Since, by $2.6, c(F)= \pm E_{r} \gamma$, it follows that to prove 0.2 (a) we have to show that

$$
p^{v} \gamma \notin i^{\prime} \pi_{m+t-1}\left(S_{r-2}^{n}\right)+Q_{r} \pi_{m+t+1}\left(S^{r n+1}\right) \quad(v<\min (s-k, w)) .
$$

Now combining 1.6 and 2.5 we obtain

$$
H j \gamma= \pm E^{(r-1) n-1}(\phi i \alpha) \circ E^{m+1} \beta^{\prime \prime}= \pm \tau
$$

and if the conditions of 0.2 (a) are satisfied then 3.5 implies that

$$
\Delta E^{3} \theta=-r E^{2} \theta=-p^{s} E^{2} \theta
$$

and hence that

$$
p^{v} \tau=p^{k+v} E^{2} \theta \notin \Delta \pi_{m+t+1}\left(S^{r n+1}\right) \quad(v<\min (w, s-k)) .
$$

3.6 follows from 3.7 and 3.8. If $\tau=p^{k} \iota_{r n}$ then $\Delta \pi_{r n+1}\left(S^{r n+1}\right)$ is generated by $p^{s} \iota_{r n}$ and the assertion of 3.6 holds for $v<s-k$. This completes the proof.

Let $\beta^{\prime \prime} \in \pi_{t-1}\left(S^{(r-1) n-1}\right)$. For our main application of $0.2(\mathrm{a})$ we need the lemma:

LEMMA 3.9. (a) $\partial \beta^{\prime * *} \mu_{r-1}=\left[\iota_{n}\right]^{r-1} \circ \beta^{\prime \prime}$, (b) $\left(h_{r-1}\right)_{*} \beta^{\prime * *} \mu_{r-1}=(-1)^{t} E \beta^{\prime \prime}$. Since $\partial \mu_{r-1}=\left[\iota_{n}\right]^{r-1}$, 3.9(a) follows from the definition of $\beta^{n * *}$. But $\left(h_{r-1}\right)_{*} \mu_{r-1}=\iota_{(r-1) n}$ and hence we obtain $3.9(\mathrm{~b})$ on applying 1.1.

4. The double suspension. Let $p$ be an odd prime. The following lemma is due to J. C. Moore [11].

LEMMA 4.1. $E^{2}:[q, 2 m-1] \rightarrow[q+2,2 m+1]$ is an isomorphism if $q<2 m p-3$ and an epimorphism if $q=2 m p-3$.

In the case $q=2 m p-3$ the kernel of $E^{2}$ is $Z_{p}$ or 0 according as the $\bmod p$ Hopf invariant is trivial or not. (See the exact sequence on p. 147 of $[15, \mathrm{II}]$.) It follows from the result of Liulevicius [9] that the former is the case for $m>1$. Let $i_{p-1}$ denote the homomorphism induced by the injection $S^{2 m} \rightarrow S_{p-1}^{2 m}$. To obtain further information Toda [14] developed a technique for studying the homomorphisms $E_{p}$ and 


$$
i_{p-1} E:[q+2 m-1,2 m-1] \rightarrow \pi_{q+2 m}\left(S_{p-1}^{2 m} ; p\right)
$$

whose composition is $E^{2}$. As we shall see this is particularly effective in the range $2 m(p-1) \leqq q<2 m\left(p^{2}-1\right)-4$.

Let $n \geqq 4$ be even and let $\Omega S_{p-1}^{n}$ be the space of loops on $S_{p-1}^{n}$ based at $*$. $S^{n-1}$ is canonically imbedded in $\Omega S_{p-1}^{n}$ and 7.8(ii) of [14] defines an isomorphism

$$
\bar{H}_{p}: \pi_{q-1}\left(\Omega S_{p-1}^{n}, S^{n-1} ; p\right) \rightarrow[q, p n-1] .
$$

Let $\Omega: \pi_{q}\left(S_{p-1}^{n}\right) \rightarrow \pi_{q-1}\left(\Omega S_{p-1}^{n}\right)$ be the natural isomorphism. Then if $m>1$ we have a sequence

$$
\stackrel{J}{\rightarrow}[q+2 m+1,2 m p-1] \stackrel{\chi}{\longrightarrow}[q+2 m-1,2 m-1] \stackrel{i_{p-1} E}{\longrightarrow} \pi_{q+2 m}\left(S_{p-1}^{2} ; p\right) \stackrel{J}{\rightarrow},
$$

where $\chi=\partial\left(\bar{H}_{p}\right)^{-1}$ and $J=\bar{H}_{p} j \Omega$, which is exact in the range $q>2 m(p-1)$ since the corresponding groups in the homotopy sequence of $\left(\Omega S_{p-1}^{2 m}, S^{2 m-1}\right)$ are finite if $q>2 m(p-1)$. Suppose that $m>1$ and $2 m(p-1)<q<2 m\left(p^{2}-1\right)-4$. Then applying 4.1 we obtain the commutative diagram

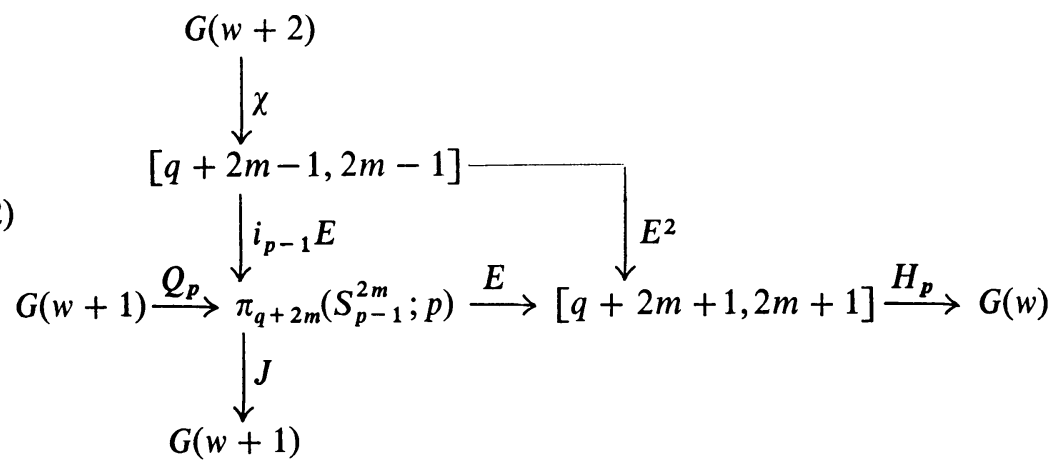

where $w=q-2 m(p-1)$, and in which $G(q)$ denotes the $p$-component of the stable group of the $q$-stem. We remark that the horizontal sequence of 4.2 applies also in the case $m=1$.

We shall assume the following information concerning the stable groups extracted from $[15,4.15]$.

LEMMA 4.3.

$$
\begin{aligned}
& G(2 t(p-1)-s)=0 \quad(1 \leqq t \leqq p, 3 \leqq s \leqq 2 p-2) ; \\
& G(2 t(p-1)-2)=0 \quad(1 \leqq t \leqq p-1) ; \quad G(2 p(p-1)-2)=Z_{p} \\
& G(2 t(p-1)-1)=Z_{p}(1 \leqq t \leqq p-1) ; \quad G(2 p(p-1)-1)=Z_{p^{2}}
\end{aligned}
$$

Proof of Theorem 0.3. We are concerned with the $p$-components of the groups of the $q$-stem (odd-dimensional spheres only) for $q<2 p(p-1)$. Thus we already know the stable $p$-component and using the result $[14,4.2]$ 


$$
\pi_{q+2}\left(S_{p-1}^{2} ; p\right)=G(q-2 p+3) \quad\left(1 \leqq q<2 p^{2}-5\right)
$$

we have a clue (via the $E-H-Q$ sequence) to $[q+3,3]$. When these extremes are trivial and the groups $G(w)$ and $G(w+1)$ of diagram 4.2 are trivial $(m \geqq 1)$, all double suspensions are epimorphisms and hence isomorphisms and all $p$-components are trivial. That such considerations yield 0.3(a) we leave to the reader. The idea of the remainder of the proof is as follows. Using $0.3(a)$ we prove trivial many Whitehead products. When the conditions of $0.2(\mathrm{a})$ are satisfied, corresponding Hopf construction elements appear in the cokernels of $E^{2}$. Thus for certain stems we are able to obtain a lower bound for the product of the orders of the successive cokernels of $E^{2}$. However, using diagram 4.2 we obtain an upper bound and when the bounds are equal all can be settled.

Consider the Whitehead products

$$
\xi_{m, t}=\left[\iota_{2 m},\left[\iota_{2 m}\right]^{p+1} \circ E^{2 m(p-1)-4} \alpha_{t}\right] \in \pi_{z}\left(S_{p-2}^{2 m}\right),
$$

where $z=2(m+t)(p-1)+2 m-3$. We first prove:

LemMA 4.6. $\xi_{m, t}=0$ if $m+t \leqq p$.

We have, by $[14,4.2]$ and $0.3(a)$, that

$$
\pi_{2(t+1)(p-1)-1}\left(S_{p-2}^{2} ; p\right) \approx G(2 t(p-1)) \quad(t \leqq p-1)
$$

and hence 4.3 implies that $\xi_{1, t}=0(1 \leqq t \leqq p-1)$. If $m>1$ we have, by $[14,4.6]$, that

$$
\begin{array}{r}
\pi_{z}\left(S_{p-2}^{2 m} ; p\right) \approx[2(m+t)(p-1)+2 m-4,2 m-1]+G(2(t+1)(p-1)-2(p-m)) \\
(m+t \leqq p) .
\end{array}
$$

Since it follows from 0.3(a) that the first summand is trivial and from 3.3 that the second is trivial, we have proved 4.6. The formulae 3.9 now enable us to apply $0.2(a)$. We need merely observe that $\tau=E^{2 m p-3} \alpha_{t}$ is of order $p$ and that $E \tau$ generates $[2 m p+2 t(p-1), 2 m p+1] \approx G(2 t(p-1)-1)$. Thus the conditions of $0.2(\mathrm{a})$ are satisfied with $w=s=1, k=0$ and it follows that the elements

$$
\beta_{m, t} \in[2(m+t)(p-1)+2 m-1,2 m+1] \quad(2 \leqq m+t \leqq p)
$$

obtained by applying the Hopf construction to maps of type

$$
\left(\iota_{2 m},\left[\iota_{2 m}\right]^{p-1} \circ E^{2 m(p-1)-4} \alpha_{t}\right)
$$

are nonzero and of filtration $p-1$.

Suppose that $1 \leqq t \leqq p-1$. We proceed to examine the groups of the $(2 t(p-1)-2)$-stem and prove

Lemma 4.7.

$$
[2 t(p-1)+2 m-1,2 m+1]= \begin{cases}0 & (m \geqq t), \\ Z_{p} & (m=1, m=t-1) .\end{cases}
$$


The case $m \geqq t$ of 4.7 follows from 4.3 for here we are in the stable range. If $m=t-1$ the remark following 4.1 implies that the kernel of $E^{2}:[2 t(p-1)+2 m-1,2 m+1] \rightarrow G(2 t(p-1)-2)=0$ is $Z_{p}$. If $m=1,4.4$ and 4.3 imply that $\pi_{2 t(p-1)}\left(S_{p-1}^{2} ; p\right)=Z_{p}$. Moreover, the image of a generator under the homomorphism

$$
E_{p}: \pi_{2 t(p-1)}\left(S_{p-1}^{2} ; p\right) \rightarrow[2 t(p-1)+1,3]
$$

has to be $\beta_{1, t}$. The required result follows from the fact that $E_{p}$ is an epimorphism which can be deduced from the vanishing of the appropriate group in the $E-H-Q$ sequence.

Lemma 4.7 includes the cases $m=1, m=t-1$ of $0.3(\mathrm{~b})(\mathrm{i})$. Let $\Gamma$ be the product of the orders of the cokernels and $K$ the product of the orders of the kernels of the homomorphisms

$$
\begin{aligned}
E^{2}:[2 t(p-1)+2 m-1,2 m+1] & \rightarrow[2 t(p-1)+2 m+1,2 m+3] \\
& (1 \leqq m \leqq t-2) .
\end{aligned}
$$

Since $\beta_{m, t-m}(2 \leqq m \leqq t-1)$ make contributions to the cokernels we have

$$
\Gamma \geqq p^{t-2} \text {. }
$$

The groups $G(w+2)$ with $w=2 t(p-1)-2, t<p$ are trivial. Thus diagram 4.2 implies that the factors $i_{p-1} E$ are monomorphisms, and, since $G(2 t(p-1)-1)=Z_{p}$, the orders of the kernels of the factors $E_{p}$ are at most $p$. Hence we have

$$
K \leqq p^{t-2}
$$

However 4.7 implies that $\Gamma=K$ and hence $\Gamma=K=p^{t-2}$. Thus the kernel and cokernel of each $E^{2}$ is $Z_{p}$. The remaining cases of $0.3(\mathrm{~b})(\mathrm{i})$ follow immediately.

We now study the $(2 p(p-1)-2)$-stem and obtain the following analogue of Lemma 4.7.

LEMMA 4.9.

$$
[2 p(p-1)+2 m-1,2 m+1]= \begin{cases}Z_{p} & (m \geqq p), \\ Z_{p^{2}} \text { or } Z_{p}+Z_{p} & (m=p-1, m=2) .\end{cases}
$$

If $m \geqq p$ we are in the stable range. The case $m=p-1$ of 4.9 follows from the fact that the kernel of $E^{2}:\left[2 p^{2}-3,2 p-1\right] \rightarrow\left[2 p^{2}-1,2 p+1\right]$ is $Z_{p}$ and hence $\left[2 p^{2}-3,2 p-1\right]$ is an extension of $Z_{p}$ by $Z_{p}$.

The case $m=2$ we postpone and remark that the assertion 0.3(b)(iv) follows from 4.9 and an argument (which we leave to the reader) exactly similar to the one given above showing that the kernels and cokernels of the intermediate double suspensions are all $Z_{p}$.

To complete the proof of 4.9 we first prove

LeMma 4.10. (a) $[2 p(p-1)+1,3]=Z_{p}=\left\{\beta_{1, p-1}\right\}$, (b) $\beta_{1, p-1}=\lambda \alpha_{1} \circ E^{2 p-3} \alpha_{p-1}$, where $\lambda \not \equiv 0(\bmod p)$. 
By 4.4 and $4.3, \pi_{2 p(p-1)}\left(S_{p-1}^{2} ; p\right) \approx G\left(2(p-1)^{2}-1\right)=Z_{p}$, which is generated by the stable suspension of $\alpha_{p-1} \cdot 4.10(\mathrm{~b})$ now follows from the fact that the isomorphism 4.4 is induced by composition with the element $\alpha^{\prime}$ of $\pi_{2 p-1}\left(S_{p-1}^{2}\right)$ such that $E_{p} \alpha^{\prime}=\alpha_{1}$. Moreover on examining the appropriate group in the $E-H-Q$ sequence we find that $E_{p}$ maps on to $[2 p(p-1)+1,3]$ which proves $4.10(\mathrm{a})$ and $0.3(\mathrm{~b})(\mathrm{iii})$.

We next observe from diagram 4.2 using our knowledge of the stable groups that

$$
i_{p-1} E:[2 p(p-1)+1,3] \rightarrow \pi_{2 p(p-1)+2}\left(S_{p-1}^{4} ; p\right)=G \text { (say) }
$$

is a monomorphism with cokernel no larger than $Z_{p}$. Since $\beta_{2, p-2}$ has filtration $p-1$ it follows that the cokernel is precisely $Z_{p}$ and hence that $G$ is an extension of $Z_{p}$ by $Z_{p}$. It only remains to prove that $E_{p}: G \rightarrow[2 p(p-1)+2,5]$ is a monomorphism (it is certaintly onto) or equivalently that the image of $Q_{p}: G(2(p-2)(p-1)-1)=Z_{p} \rightarrow G$ is the zero of $G$. Now the iterated suspension of $\alpha_{p-2}$ generates $G(2(p-2)(p-1)-1)$. Thus, by Lemma 3.3, it will be sufficient to prove:

LEMMA 4.11. $\left[\iota_{4}\right]^{p} \circ E^{4 p-4} \alpha_{p-2}=0 \in G$.

Suppose 4.11 is false. Then 4.10 (b) implies that $E^{2} \alpha_{1} \circ E^{2 p-1} \alpha_{p-1}=0$. Thus the toric element

$$
\gamma=\left\{E^{2} \alpha_{1}, E^{2 p-1} \alpha_{p-1}, p \iota_{k+3}\right\} \in \pi_{k+4}\left(S^{5}\right),
$$

where $k=2 p(p-1)$, is defined. Moreover by $[15,4.17$ (ii)] its stable suspension is a generator of $G(k-1)=Z_{p^{2}}$, which implies that the order of $\gamma$ is at least $p^{2}$. Now $[k+2,3]$ contains the nonzero element $\alpha_{p}$ and since $\pi_{k+1}\left(S_{p-1}^{2} ; p\right)=0$ and $[k+2,2 p+1]=Z_{p}$ it follows that $[k+2,3]=Z_{p}=\left\{\alpha_{p}\right\}$. On examining the relevant groups in 4.2 we find that $i_{p-1} E:[k+2,3] \rightarrow \pi_{k+3}\left(S_{p-1}^{4} ; p\right)$ is an isomorphism and hence that $\pi_{k+3}\left(S_{p-1}^{4} ; p\right)=Z_{p}$. We can conclude that

$$
H_{p} \gamma \neq 0 \in[k+4,4 p+1]=\left\{E^{4 p-2} \alpha_{p-2}\right\}
$$

and hence that $Q_{p} E^{4 p-2} \alpha_{p-2}=\left[\iota_{4}\right]^{p} \circ E^{4 p-4} \alpha_{p-2}=0$, which contradicts the assumption. The proof of 4.9 and $0.3(\mathrm{~b})$ is now complete.

It only remains to prove $0.3(\mathrm{c})$. We have already seen that $[2 t(p-1)+2,3]=Z_{p}$ in the case $t=p$. The case $1<t<p$ which is similar we leave to the reader and the result is well known if $t=1.0 .3$ (c)(i) and (ii) may be deduced from 4.3 and the lemma:

LEMMA 4.12. If $t=p$ and $m \geqq 3$ or if $t<p$ and $m \geqq 2$ then $E^{2}:[2 t(p-1)+2 m-2,2 m-1] \rightarrow[2 t(p-1)+2 m, 2 m+1]$ is an isomorphism.

If $m>t, 4.12$ follows from 4.1. If $m \leqq t$ we may refer to diagram 4.2 from which we easily obtain that the factors $i_{p-1} E$ are isomorphisms and that the 
factors $E_{p}$ are monomorphisms. However in our study of the $(2 t(p-1)-2)$-stems $(t \leqq p)$ we saw that the kernel of

$$
E_{p}: \pi_{2 t(p-1)+2 m-2}\left(S_{p-1}^{2 m} ; p\right) \rightarrow[2 t(p-1)+2 m-1,2 m+1]
$$

is $Z_{p}$ if $t<p$ and $2 \leqq m \leqq t-1$ or if $t=p$ and $3 \leqq m \leqq p-1$. Since $G(2(t-m)(p-1)-1)=Z_{p}$, the exactness of the $E-H-Q$ sequence implies that the corresponding homomorphisms $Q_{p}$ are nontrivial and that the $E_{p}$ factors in the $(2 t(p-1)-1)$ stems are epimorphisms and hence isomorphisms, which proves 4.12 .

\section{BIBLIOGRAPHY}

1. A. L. Blakers and W. S. Massey, Products in homotopy theory, Ann. of Math. (2) 58 (1953), 295-324.

2. K. A. Hardie, Note on the Hopf-James invariants, Proc. Cambridge Philos. Soc. 57 (1961), 746-753.

3. - A generalization of the Hopf construction, Quart. J. Math. Oxford Ser. (2) 12 (1961), 196-204.

4. - Higher Whitehead products, Quart. J. Math. Oxford Ser. (2) 12 (1961), 241-249.

5. P. J. Hilton, Suspension theorems and the generalized Hopf invariant, Proc. London Math. Soc. (3) 1 (1951), 462-493.

6. I. M. James, Reduced product spaces, Ann. of Math. (2) 62 (1955), 170-197.

7. - On the suspension triad, Ann. of Math. (2) 63 (1956), 191-247.

8. - Filtration of the homotopy groups of spheres, Quart. J. Math. Oxford Ser. (2) 9 (1958), 301-309.

9. A. Liulevicius, The factorization of cyclic reduced powers, Proc. Nat. Acad. Sci. U.S.A. 46 (1960), 978-981.

10. W. S. Massey, Exact couples in algebraic topology. I, II, Ann. of Math. (2) 56 (1952), 363-396.

11. J. C. Moore, Some applications of homology theory to homotopy problems, Ann. of Math. (2) 58 (1953), 325-350.

12. E. H. Spanier, Duality and the suspension category, pp. 259-272, Symposium Internacional de Topologia Algebraica, Unesco, 1958.

13. H. Toda, Generalised Whitehead products and homotopy groups of spheres, J. Inst. Polytech. Osaka City Univ. Ser. A 3 (1952), 43-82.

14. - On the double suspension $E^{2}$, J. Inst. Polytech. Osaka City Univ. Ser. A 7 (1956), $103-145$.

15. - p-Primary components of homotopy groups. IV, Mem. Coll. Sci. Univ. Kyoto Ser. 32 (1959), 297-332.

16. - On unstable homotopy of spheres and classical groups, Proc. Nat. Acad. Sci. U.S.A. 46 (1960) 1102-1105.

17. G. W. Whitehead, A generalization of the Hopf invariant, Ann. of Math. (2) 51 (1950), 192-237.

Wayne State University,

Detroit, Michigan

UNIVERSITY OF CAPE TOWN,

Cape Town, South Africa 